\title{
INVARIANT COMPLEMENTS NORMALIZED BY THE FIXED POINT SUBGROUP
}

\author{
ANTONIO BELTRÁN \\ DEPARTAMENTO DE MATEMÁTICAS \\ UNIVERSITAT JAUME I \\ 12071 CASTELLÓN, SPAIN
}

\begin{abstract}
Let $A$ and $G$ be finite groups and assume that $A$ acts on $G$ by automorphisms with $(|A|,|G|)=1$. Let $\mathbf{C}_{G}(A)$ be the fixed point subgroup and fix a prime number $p$. We prove that if $G$ possesses an $A$-invariant $p$-complement that is normalized by $\mathbf{C}_{G}(A)$, then $G$ is $p$-solvable. We also extend Frobenius criterion for $p$-nilpotency, in the coprime action setting, for the primes 2 and 3 .
\end{abstract}

\section{INTRODUCTION}

Let $A$ and $G$ be finite groups with $(|A|,|G|)=1$ such that $A$ acts by automorphisms on $G$. Let denote by $C:=\mathbf{C}_{G}(A)$ the fixed point subgroup. In the coprime action scenario, quite a few research works seem to confirm that $A$-invariant subgroups of $G$ that are normalized by $C$ may take on the role of normal subgroups (see for instance [1], [2]). An elementary first property revealing this fact is that $G$ has exactly one $A$-invariant Sylow $p$-subgroup for certain prime $p$ if and only if it is normalized by $C$. Our purpose is to generalize the celebrated Frobenius criterion for $p$-nilpotency, which claims that a group is $p$-nilpotent, that is, has a normal Hall $p^{\prime}$-subgroup, if and only if every local subgroup (the normalizer of a non-trivial $p$-subgroup) of $G$ is $p$-nilpotent. Thus, we are looking for a "local" $A$-version that ensures the existence of $A$-invariant $p$-complement normalized by $C$.

The existence of normal $p$-complement obviously implies $p$-solvability, and our first result, Theorem A, goes in this direction. Its proof is based on the Classification of Finite Simple Groups, more precisely, on Guralnick's classification of prime power index subgroups of simple groups [5]. We remark that the existence of $A$-invariant $p$-complement normalized by $C$ in a group $G$ easily leads to the $p$-nilpotency of $C$, however, only imposing this latter hypothesis does not imply the $p$-solvability of $G$. We will provide examples throughout the proofs.

2010 Mathematics Subject Classification. 20E28, 20D15, 20D06, 20 D45.

Key words and phrases. Coprime action, normal $p$-complements, Frobenius $p$-nilpotency criterion, Finite simple groups.

Antonio Beltrán: abeltran@uji.es . 
Theorem A. Let $A$ and $G$ be finite groups with $(|A|,|G|)=1$ and assume that $A$ acts on $G$ by automorphisms. Let $p$ be a fixed prime. If $G$ has an $A$-invariant $p$-complement normalized by $\mathbf{C}_{G}(A)$, then $G$ is p-solvable. As a consequence, such A-invariant p-complement is unique.

We utilize Theorem A to obtain our next contribution, which partially extends the $p$-nilpotency criterion of Frobenius.

Theorem B. Let $A$ and $G$ be finite groups with $(|A|,|G|)=1$ and assume that $A$ acts on $G$ by automorphisms. Let $C:=\mathbf{C}_{G}(A)$ and $p$ a prime. Assume that for every A-invariant p-subgroup $1 \neq P$ of $G$, the group $\mathbf{N}_{G}(P)$ has an $A$-invariant $p$-complement normalized by $C \cap \mathbf{N}_{G}(P)$. If $G$ is $p$-solvable, or if $p=2$ or 3 , then $G$ has an $A$-invariant $p$-complement normalized by $C$.

Precisely, Theorem A is used to reduce the proof of Theorem B to the non-abelian simple case, and then a detailed analysis on coprime action on these groups is required. The fact that all except one family of simple groups (Suzuki simple groups), acted on by a coprime automorphism group, satisfy that the fixed point subgroup $C$ has order divisible by 6 allows to reduce our study to a few families of simple groups of Lie type whose fixed point subgroup $C$ has a specific structure. Then, the subgroup structure of those families of simple groups is needed. For primes distinct from 2 and 3, however, Theorem B is false in general and we will provide examples of it.

We also want to note that a complete although weaker extension of the $p$-nilpotency theorem of Frobenius has appeared in [10]. It concerns, however, with the existence of normal $p$-complement instead of $p$-complement normalized by $C$. Theorem $\mathrm{B}$ of $[10]$ establishes that, in the coprime action context, a group $G$ is $p$-nilpotent when $\mathbf{N}_{G}(P)$ is $p$-nilpotent for every non-trivial invariant $p$-subgroup $P$ of $G$.

A priori, the existence of $A$-invariant $p$-complement is not inherited by $A$-invariant subgroups, but Theorem A implies that this is true when such a $p$-complement is normalized by $C$. As a consequence, the reciprocal of Theorem B holds for every finite group, and we will give a proof (Corollary 3.3). All groups are supposed to be finite and the notation is standard as in [9].

\section{Preliminaries}

In this section we collect the main results that we need in the proofs. The first theorem, which has been pointed out in the introduction, relays on the Classification of the Finite Simple Groups and is essential in our approach on the $p$-complements in non-abelian simple groups for proving Theorem A.

Theorem 2.1. [5, Theorem 1] Let $G$ be a non-abelian simple group with $H<G$ and $|G: H|=p^{a}$, $p$ prime. One of the following holds.

(a) $G=A_{n}$, and $H \cong A_{n-1}$, with $n=p^{a}$.

(b) $G=\operatorname{PSL}(n, q)$ and $H$ is the stabilizer of a line or hyperplane. Then $|G: H|=$ $\left(q^{n}-1\right) /(q-1)=p^{a}$.

(c) $G=\operatorname{PSL}(2,11)$ and $H \cong A_{5}$.

(d) $G=M_{23}$ and $H \cong M_{22}$ or $G=M_{11}$ and $H \cong M_{10}$. 
(e) $G=\operatorname{PSU}(4,2) \cong \operatorname{PSp}(4,3)$ and $H$ is a subgroup of index 27.

Notice that the corresponding subgroups in the above theorem are not necessarily $p$ complements of $G$. In order to get more information on case (b), we will make use of the renowned Zsigmondy's property.

Lemma 2.2. [13] Let $q$ be a natural number greater than 1. Then for every natural number $m$ there exists a (Zsigmondy) prime $r$ such that $r \mid q^{m}-1$ but $r \nmid q^{i}-1$ for every $1 \leq i \leq m-1$ except for the following cases:

(1) $m=6$ and $q=2$;

(2) $m=2$ and $q=2^{k}-1$ for some natural number $k$.

As a consequence of Lemma 2.2, it is not difficult to prove (for instance, by using the solved Catalan's conjecture [12]) the following properties.

Lemma 2.3. [5, (3.3)] Suppose that $q=r^{b}$, with $r$ prime and $b \geq 1$ and that $\left(q^{n}-\right.$ 1) $/(q-1)=p^{a}$, with $p$ prime. Then

(1) $n$ is prime;

(2) $r \equiv 1(\bmod n)$ or $n=r=2$;

(3) If $n=2$ then either $q$ is a Mersenne prime and $p=2$; $a=1$ and $p$ is a Fermat prime; or $a=2, p=3$ and $q=8$.

With regard to coprime action, we recall the following. Suppose that a group $A$ acts coprimely on a group $G$. Then, for every prime $p$, there always exists an $A$-invariant Sylow $p$-subgroup in $G$ and any two of them are conjugate by some element lying in $C$. Furthermore, if $P$ is an $A$-invariant Sylow subgroup of $G$, then $P \cap C$ is a Sylow subgroup of $C$. The same properties hold for $A$-invariant $p$-complements when $G$ is $p$-solvable. We refer to [11, Chapter 8] or [9, Chapter 3] for a detailed presentation and basic properties of coprime action. We only state here two known results.

Lemma 2.4. $[11,8.2 .2]$ Let $A$ be a group that acts on a group $G$. Let $N$ be an $A$ invariant normal subgroup of $G$. Suppose that the action of $A$ on $N$ is coprime. Then $\mathbf{C}_{G / N}(A)=\mathbf{C}_{G}(A) N / N$.

The proof of the following result, which concerns with action on direct products, however, does not require the coprimeness hypothesis.

Lemma 2.5. [11, 8.1.6] Suppose that a finite group $A$ acts on a finite group $G$ which allows a direct decomposition $G=H_{1} \times \cdots \times H_{n}$, that is $A$-invariant under $A$, that is, $H_{i}^{a} \in\left\{H_{1}, \ldots, H_{n}\right\}$ for all $a \in A$ and all $i \in\{1, \ldots, n\}$. Assume further that $A$ acts transitively on $\left\{H_{1}, \ldots, H_{n}\right\}$. Let $H \in\left\{H_{1}, \ldots, H_{n}\right\}$, let $B=\mathbf{N}_{A}(H)$, and let $S$ be a transversal for the cosets of $B$ in $A$. Then

(a) $\mathbf{C}_{G}(A)=\left\{\Pi_{s \in S} e^{s} \mid e \in \mathbf{C}_{H}(B)\right\}$;

(b) $\mathbf{C}_{G}(A) \cong \mathbf{C}_{H}(B)$.

For proving Theorem B, we make use of the structure of the Sylow normalizers in certain families of simple groups of Lie type, so we have summarized them for the reader's convenience.

Lemma 2.6. Let $G=\operatorname{PSL}(2, q)$, where $q$ is a power of prime $r$ and $d=(2, q+1)$. Let $p$ be a prime divisor of $|G|$ and $P$ a Sylow p-subgroup of $G$. 
(1) If $p=r$, then $\mathbf{N}_{G}(P)=P \rtimes C_{\frac{q-1}{d}}$.

(2) If $2 \neq p \mid \frac{q+1}{d}$, then $\mathbf{N}_{G}(P)=C_{\frac{q+1}{d}} \rtimes C_{2}$;

(3) If $2 \neq p \mid \frac{q-1}{d}$, then $\mathbf{N}_{G}(P)=C_{\frac{q-1}{d}} \rtimes C_{2}$;

(4) Assume $r \neq p=2$.

(4.1) If $q \equiv \pm 1(\bmod 8)$, then $\mathbf{N}_{G}(P)=P$;

(4.2) If $q \equiv \pm 3(\bmod 8)$, then $\mathbf{N}_{G}(P)=\left(C_{2} \times C_{2}\right) \rtimes C_{3}$.

Proof. This follows from [8, Chapter II, Theorem 8.27].

Lemma 2.7. Let $p$ be a prime divisor of the order of $G=\operatorname{Sz}(q)$, where $q=2^{2 n+1}$ and $n \geq 1$, and let $P$ be a Sylow p-subgroup of $G$.

(1) If $p=2$, then $\mathbf{N}_{G}(P)=P \rtimes C_{q-1}$;

(2) If $p \mid(q-1)$, then $\mathbf{N}_{G}(P)=D_{2(q-1)}$;

(3) If $p \mid(q \pm \sqrt{2 q}+1)$, then $\mathbf{N}_{G}(P)=C_{q \pm \sqrt{2 q}+1} \rtimes C_{4}$.

Proof. This follows from [7, Chapter XI, Theorem 3.10].

Lemma 2.8. Let $p$ be a prime divisor of the order of $G=\operatorname{PSU}(3, q)$, where $q=2^{r}$ and $r \geq 2$, and let $P$ be a Sylow p-subgroup of $G$ and $d=(3, q+1)$.

(1) If $p=2$, then $\mathbf{N}_{G}(P)=P \rtimes C_{\frac{q^{2}-1}{d}}$;

(2) If $p \mid(q+1)$, then $\mathbf{N}_{G}(P)=\left(C_{\frac{q+1}{d}} \times C_{q+1}\right) \rtimes S_{3}$;

(3) If $p \mid(q-1)$, then $\mathbf{N}_{G}(P)=C_{\frac{q+1}{d}} \times D_{2(q-1)}$;

(4) If $p \mid\left(q^{2}-q+1\right)$ and $p \neq 3$, then $\mathbf{N}_{G}(P)=\left(C_{\left(q^{2}-q+1\right) / d}\right) \rtimes C_{3}$.

Proof. This follows from [6].

We end this section by listing the groups of Lie type that are non-simple. This can be found in [3] for instance.

Remark 2.9. The finite groups of Lie type that are non-simple are the following:

(i) four solvable groups: $A_{1}(2) \cong \operatorname{PSL}(2,2) \cong S_{3} ; A_{1}(3) \cong \operatorname{PSL}(2,3) \cong A_{4} ;{ }^{2} A_{2}(2) \cong$ $\operatorname{PSU}(3,2)$, which is a Frobenius group with complement $Q_{8}$; and ${ }^{2} B_{2}(2) \cong \mathrm{Sz}(2)$, the Frobenius group of order 20;

(ii) four non-solvable groups: $B_{2}(2) \cong S_{6} ; G_{2}(2)$, of order 12096 and whose derived subgroup is isomorphic to $\operatorname{PSU}(3,3) ;{ }^{2} G_{2}(3)$, whose derived subgroup is isomorphic to $\operatorname{PSL}\left(2,2^{3}\right)$; and ${ }^{2} F_{4}(2)$, whose derived subgroup is the Tits (simple) group.

\section{Main Results}

For proving Theorem A, we induct on the order of $G$, and we reduce it to show that a non-abelian simple group acted on coprimely by a group $A$ cannot possess any $A$-invariant $p$-complement normalized by $\mathbf{C}_{G}(A)$ for any prime $p$ dividing $|G|$. We state and prove this result separately.

Theorem 3.1. Let $G$ be a non-abelian simple finite group and suppose that a group $A$ acts coprimely on $G$. Let $p$ be a fixed prime. If $G$ has an A-invariant p-complement normalized by $\mathbf{C}_{G}(A)$, then $G$ is a $p^{\prime}$-group. 
Proof. Let $p$ be a prime dividing $|G|$ and suppose that $G$ has an $A$-invariant $p$-complement normalized by $\mathbf{C}_{G}(A)$. The theorem will be proved if we achieve a contradiction. Since such $p$-complement has index a power of $p$, in particular, $G$ should be isomorphic to one of the groups appearing in Lemma 2.1 (and $p$ should be the corresponding prime in each case). However, it is well-known that all groups appearing in that list except those of case (b), that is, when $G$ is isomorphic to $A_{n}$ with $n \geq 5, \operatorname{PSL}(2,11), \operatorname{PSU}(4,2)$ or the sporadic simple groups $M_{11}$ and $M_{23}$, do not admit non-trivial coprime automorphisms. In fact, from [3, Table 5] the only non-abelian simple groups which admit a non-trivial coprime action are simple groups of Lie type defined over some finite field (indeed, it turns out that $A / \mathbf{C}_{A}(G)$ is a group of automorphisms of the underlying field). This forces $A$ to act trivially on any of them, but certainly $G$ cannot have normal $p$-complement, against the hypotheses. Therefore, we will assume that $G \cong \operatorname{PSL}(n, q)$, for some integer $n$ and some prime power $q$, say $q=r^{d}$, with $r$ prime. Furthermore, Lemma 2.1(b) establishes

$$
\frac{q^{n}-1}{(q-1)}=p^{a} \text { for some } a \geq 1 \text {. }
$$

We will prove that $A$ acts trivially in this case too, and this will give rise again to a contradiction. Recall that the order of the Singer cycle of $\operatorname{PSL}(n, q)$ is equal to

$$
\frac{q^{n}-1}{(n, q-1)(q-1)}=\frac{p^{a}}{(n, q-1)} \text {. }
$$

This yields two possibilities: $(n, q-1)=p^{t}$ with $t>1$, or $(n, q-1)=1$. Suppose first that $(n, q-1)=p^{t}$. Assume further that $n=2$ and hence, $p=2$. Then by Lemma $2.3(3)$, we have that $q$ is a Mersenne prime, and consequently, the action of $A$ on $G$ is trivial, as wanted. We may suppose then that $n \geq 3$. By Lemma 2.2, there is a prime dividing $q^{n}-1$ and not dividing $q-1$. This prime must be $p$, contradicting the fact that $p$ divides $q-1$. Hence, we may assume the second possibility, that is, $(n, q-1)=1$. By applying Lemma 2.3(2), we have either $r \equiv 1(\bmod n)$ or $n=r=2$. The first case trivially implies that $n \mid q-1$, contradicting our assumption. So $n=r=2$, and again by Lemma 2.3(3), we get either $p^{a}=9$ and $q=8$, or $p=2^{d}+1$ is a Fermat prime, which forces $d$ to be a power of 2 . In the former case, $G=\operatorname{PSL}(2,8)$, which does not admit non-trivial coprime action, so we are finished. In the latter case, $G=\operatorname{PSL}\left(2,2^{d}\right)$ with $d$ a power of 2 . But it is known that $\operatorname{Out}(G) \cong C_{d}$, and accordingly, $G$ does not have non-trivial coprime automorphisms either. This finishes the proof.

We are ready to prove Theorem A.

Proof of Theorem $A$. We argue by induction on $|G|$ and write $C:=\mathbf{C}_{G}(A)$. Let $N$ be an $A$-invariant normal subgroup of $G$. First, we prove that $N$ and $G / N$ satisfy the hypothesis of the theorem. Indeed, if $K$ is an $A$-invariant $p$-complement of $G$ that is normalized by $C$, then $K \cap N$ clearly is an $A$-invariant $p$-complement of $N$ normalized by $N \cap C$, and likewise, $K N / N$ is an $A$-invariant $p$-complement of $G / N$ normalized by $C N / N=\mathbf{C}_{G / N}(A)$ (this equality is given in Lemma 2.4). Hence if $N<G$, then the inductive hypothesis implies that $N$ and $G / N$ are $p$-solvable, and consequently, so is $G$.

Thus, we can assume that $G$ does not have any proper and non-trivial $A$-invariant normal subgroup, so we write $G=H_{1} \times \ldots \times H_{n}$, where $H_{i}$ are isomorphic non-abelian simple groups. Furthermore, we know that $A$ acts transitively on $\left\{H_{i}\right\}_{i=1}^{n}$. Assume that 
$n>1$, let $B=\mathbf{N}_{A}(H)$ where $H=H_{1}$, and let $S=\left\{1=s_{1}, s_{2}, \ldots, s_{n}\right\}$ be a transversal for the cosets of $B$ in $A$. Let us choose $K$ an $A$-invariant $p$-complement of $G$ normalized by $C$ given by the hypothesis. Observe that $K=K \cap H_{1} \times \ldots \times K \cap H_{n}$, where $K \cap H_{i}$ is a $p$-complement of $H_{i}$ for every $i$. Also, $K \cap H$ is $B$-invariant. By using Lemma 2.5, we next prove that $K \cap H$ is normalized by $\mathbf{C}_{H}(B)$. Indeed, if $e \in \mathbf{C}_{H}(B)$, then we write $c=\prod_{i=1}^{n} e^{s_{i}} \in \mathbf{C}_{G}(A)$, and since $e^{s_{i}} \in H^{s_{i}} \neq H$ centralizes $H$ for every $i=2, \ldots, n$, we obtain

$$
(K \cap H)^{e}=(K \cap H)^{c}=K^{c} \cap H^{c}=K \cap H
$$

as wanted. Thus, by induction, $H$ is $p$-solvable (indeed, the simplicity forces $H$ to be a $p^{\prime}$-group), and we are finished. Therefore, $G$ must be non-abelian simple. By Theorem 3.1 , we conclude that $G$ is a $p^{\prime}$-group, so in particular, $G$ is $p$-solvable. The uniqueness of the statement is an immediate consequence of the $p$-solvability of $G$ and Glauberman's Lemma [9, Lemma 3.24].

We can prove now Theorem B, which we state again as follows.

Theorem 3.2. Let $A$ be a group that acts on a finite group $G$ with $(|A|,|G|)=1$. Let $C:=\mathbf{C}_{G}(A)$ and $p$ a prime. Assume that for every $A$-invariant $p$-subgroup $1 \neq P$ of $G$, the group $\mathbf{N}_{G}(P)$ has an $A$-invariant $p$-complement normalized by $\mathbf{N}_{C}(P)$.

(a) If $G$ is p-solvable, then $G$ has an A-invariant $p$-complement normalized by $C$.

(b) If $p=2$ or 3 , then $G$ has an A-invariant $p$-complement normalized by $C$.

Proof. We proceed by induction on the order of $G$ to prove both (a) and (b). Suppose that $N$ is a minimal $A$-invariant normal subgroup of $G$ and we see that $N$ satisfies the hypothesis. Indeed, let $1 \neq P$ be an $A$-invariant $p$-subgroup of $N$. Since $\mathbf{N}_{G}(P)$ has an $A$-invariant $p$-complement normalized by $C \cap \mathbf{N}_{G}(P)$, it certainly follows that $\mathbf{N}_{N}(P)$ has an $A$-invariant $p$-complement normalized by $C \cap \mathbf{N}_{N}(P)$. Therefore, if we assume $N<G$, by induction $N$ has an $A$-invariant $p$-complement normalized by $C \cap N$, so by Theorem A, $N$ is $p$-solvable. By minimality, there exist two possibilities for $N$ : it is a $p$-group or a $p^{\prime}$-group. If $N$ is a $p$-group, then the theorem trivially follows, so we can assume that $N$ is a $p^{\prime}$-group. We claim that $G / N$ also satisfies the hypothesis of the theorem. Let $H / N$ be a non-trivial $A$-invariant $p$-subgroup of $G / N$. Then, we can write $H=Q N$ for some $A$-invariant $p$-subgroup $Q$ of $H$ by using the Schur-Zassenhaus theorem [11, 6.2.1] and Glauberman's Lemma. By hypothesis, $\mathbf{N}_{G}(Q)$ has an $A$-invariant $p$-complement, say $K$, normalized by $\mathbf{N}_{C}(Q)$, and then $K N / N$ is an $A$-invariant $p$-complement of

$$
\mathbf{N}_{G}(Q) N / N=\mathbf{N}_{G / N}(Q N / N)
$$

normalized by $\mathbf{N}_{C}(Q) N / N$. Now, by applying Lemma 2.4,

$$
\mathbf{C}_{G / N}(A) \cap \mathbf{N}_{G / N}(Q N / N)=C N / N \cap \mathbf{N}_{G}(Q) N / N=\left(C N \cap \mathbf{N}_{G}(Q) N\right) / N
$$

and by applying [9, 3E.2, p. 106], we also have

$$
C N \cap \mathbf{N}_{G}(Q) N=\left(C \cap \mathbf{N}_{G}(Q) N\right) N=\left(C \cap \mathbf{N}_{G}(Q)\right)(C \cap N) N=\mathbf{N}_{C}(Q) N .
$$

Therefore, we conclude that $\mathbf{N}_{G / N}(Q N / N)$ has an $A$-invariant $p$-complement normalized by $\mathbf{C}_{G / N}(A) \cap \mathbf{N}_{G / N}(Q N / N)$, so $G / N$ satisfies the hypotheses of the theorem, as claimed. Thus, by induction, $G / N$ has an $A$-invariant $p$-complement $K / N$ normalized 
by $\mathbf{C}_{G / N}(A)=C N / N$. Since $N$ is a $p^{\prime}$-group, we conclude that $K$ is an $A$-invariant $p$-complement of $G$ normalized by $C$. Therefore, if $G$ possesses an $A$-invariant normal proper subgroup $N \neq 1$, then (a) and (b) are true. So we can assume that $G$ has no such subgroups, that is, $G$ is the direct product of isomorphic simple groups. Now, if $G$ is $p$-solvable, then $G$ is a $p$-group or a $p^{\prime}$-group, and hence (a) is proved.

Thus, to prove (b) we can assume $G=H_{1} \times \ldots \times H_{n}$, where $H_{i}$ are isomorphic nonabelian simple groups. Also, $A$ acts transitively on the set $\left\{H_{i}\right\}_{i=1}^{n}$. Assume that $n>1$, let $B=\mathbf{N}_{A}(H)$ where $H=H_{1}$, and let $S=\left\{1=s_{1}, s_{2}, \ldots, s_{n}\right\}$ be a transversal of $B$ in $A$. Next we prove that $H$, which is acted on by $B$, satisfies the hypotheses of the theorem. Without loss of generality we can assume $H^{s_{i}}=H_{i}$ for $i=1, \ldots, n$. Let $1 \neq P_{1}$ be a $B$-invariant $p$-subgroup of $H$ and put $P=\prod_{i=1}^{n} P_{1}^{s_{i}}$, which is an $A$-invariant $p$-subgroup of $G$. We know that

$$
\mathbf{N}_{G}(P)=\mathbf{N}_{G}\left(\prod_{i=1}^{n} P_{1}^{s_{i}}\right)=\prod_{i=1}^{n} \mathbf{N}_{H_{i}}\left(P_{1}^{s_{i}}\right)=\prod_{i=1}^{n} \mathbf{N}_{H}\left(P_{1}\right)^{s_{i}}
$$

and by hypothesis, $\mathbf{N}_{G}(P)$ has an $A$-invariant $p$-complement, say $K$, that is normalized by $\mathbf{N}_{C}(P)$. Also, $K \cap H$ is a $B$-invariant $p$-complement of $\mathbf{N}_{H}\left(P_{1}\right)$ (in fact, $K=\prod_{i=1}^{n} K \cap H_{i}$ ). Now, let $e \in \mathbf{C}_{H}(B) \cap \mathbf{N}_{H}\left(P_{1}\right)$. By Lemma 2.5, we have $c=\prod_{i=1}^{n} e^{s_{i}} \in \mathbf{C}_{G}(A)$ and we easily see that $c \in \mathbf{N}_{G}(P)$ by using the above equalities on normalizers. Also,

$$
(K \cap H)^{e}=(K \cap H)^{c}=K^{c} \cap H^{c}=K \cap H,
$$

so this proves that $\mathbf{N}_{H}\left(P_{1}\right)$ has a $B$-invariant $p$-complement normalized by $\mathbf{C}_{H}(B) \cap$ $\mathbf{N}_{H}\left(P_{1}\right)$. This proves that $H$ satisfies the hypothesis of the theorem. By induction, $H$ has a $B$-invariant $p$-complement normalized by $\mathbf{C}_{H}(B)$, say $T$. Again by Lemma 2.5, it is clear that $\prod_{i=1}^{n} T^{s_{i}}$ is an $A$-invariant $p$-complement of $G$ normalized by $C$, and the theorem is proved.

So for the rest of the proof we may assume that $G$ is non-abelian simple (of order divisible by $p$ ). Furthermore, if the action of $A$ were trivial, then Frobenius $p$-nilpotency theorem would imply that $G$ is $p$-nilpotent, which is impossible. Moreover, there is no loss if we assume that $A$ acts faithfully on $G$, otherwise we replace $A$ by $A / \mathbf{C}_{A}(G)$, that is, we can assume that $A \leq \operatorname{Aut}(G)$. As alternating groups and sporadic simple groups do not admit non-trivial coprime action (see [3, Table 5] for instance), we assume that $G$ is a simple group of Lie type, say $\mathrm{G}(q)$, over a field of $q$ elements, with $q$ a prime power. The structure of $\operatorname{Aut}(G)$ is described, for instance, in [4, Theorem 2.5.1], and it follows that $A$ is (conjugate to) some group of field automorphisms of $G$. Thus, by replacing $A$ by some conjugate, we get that $A$ is cyclic, say of order $r$. In the general case, it is known that the centralizer of a field automorphism of a group of Lie type $\mathrm{G}(q)$ is an intermediate subgroup between $\mathrm{G}\left(q_{0}\right)$, the group of Lie type of the same type as $G$ but defined over a field of $q_{0}$ elements where $q_{0}^{r}=q$, and its group of inner-diagonal automorphisms. From now on we distinguish the two possibilities for $p$ given in the statement, and we will seek for a contradiction, which will prove the theorem.

Suppose first that $p=2$. As we have said in the above paragraph, $C$ contains a group of Lie type $\mathrm{G}\left(q_{0}\right)$, so by the list given in Remark 2.9 , we know that 2 divides the order of the fixed point subgroup for every simple group of Lie type. Moreover, since every 2 -subgroup of $C$ is $A$-invariant, then the hypothesis and the Frobenius $p$-nilpotency theorem certainly 
imply that $C$ is 2-nilpotent, so in particular, $\mathrm{G}\left(q_{0}\right)$ must be 2-nilpotent too, and by taking into account Remark 2.9, the only possibilities for $\mathrm{G}\left(q_{0}\right)$ are: $\operatorname{PSL}(2,2), \operatorname{PSU}(3,2)$ and $\mathrm{Sz}(2)$. We analyze them separately.

Assume first that $G=\operatorname{PSL}\left(2,2^{r}\right)$ and $\mathrm{G}\left(q_{0}\right) \cong \operatorname{PSL}(2,2)$. In this case, $\operatorname{Aut}(\operatorname{PSL}(2,2))=$ $\operatorname{PSL}(2,2)$, so $C \cong \operatorname{PSL}(2,2)$. Then we take $P$ to be an $A$-invariant Sylow 2 -subgroup of $G$. By Lemma 2.6(1), $\mathbf{N}_{G}(P)=P \rtimes C_{2^{r}-1}$. Also, by hypothesis, $\mathbf{N}_{G}(P)$ has an $A$-invariant 2complement, say $H$, which is normalized by $\mathbf{N}_{C}(P)$. Since $C_{2} \cong P \cap C \leq \mathbf{N}_{C}(P)$, then in particular, $[H, P \cap C] \subseteq H \cap P=1$, that is, $P \cap C$ centralizes $H$. However, by considering orders we observe that $H$ is a Hall subgroup of $G$, and if we choose any $A$-invariant Sylow subgroup of $H$, then by Lemma 2.6(3), we get that $H$ cannot be centralized by $P \cap C$. This contradiction shows that this case is not possible.

Assume now that $G=\mathrm{Sz}\left(2^{r}\right)$ and $\mathrm{G}\left(q_{0}\right) \cong \mathrm{Sz}(2)$. Since the outer automorphism group of the Suzuki simple group is cyclic, given by field automorphisms, we have that $\mathrm{Sz}(2)$ has no non-inner inner-diagonal automorphism, and hence $C \cong \mathrm{Sz}(2)$. Then we choose $P$ to be an $A$-invariant Sylow 2-subgroup of $G$. By Lemma 2.7(1), we know that $\mathbf{N}_{G}(P)=P \rtimes C_{2^{r}-1}$, has an $A$-invariant 2-complement, say $H$, normalized by $\mathbf{N}_{C}(P)$. Arguing as in the above case, we obtain that $C_{4} \cong P \cap C$ centralizes $H$. Moreover, by taking orders, $H$ is a Hall subgroup of $G$, so we may choose an $A$-invariant Sylow subgroup of $G$ contained in $H$. By applying Lemma 2.7(2), such Sylow subgroup cannot be centralized by $P \cap C$. Therefore, this case is not possible either.

Finally, assume that $G=\operatorname{PSU}\left(3,2^{r}\right)$ and $\mathrm{G}\left(q_{0}\right) \cong \operatorname{PSU}(3,2)$. As its group of automorphisms is $\operatorname{PGU}(3,2)=3^{2}: \operatorname{GL}(2,3)$ and this is not 2-nilpotent, it follows that the unique possibility for $C$ is $\operatorname{PSU}(3,2)$. The arguments to discard this case are similar. It is enough to take $P$ a Sylow 2-subgroup of $G$, which in this case satisfies $P \cap C \cong Q_{8}$, and then apply Lemma 2.8(1),(2) and (3) to get a contradiction. This concludes the proof for $p=2$.

We prove now (b) for $p=3$. As above, by using Remark 2.9 and the fact that the Suzuki simple groups are the only simple groups whose order is coprime to 3 , we have that $|C|$ is always divisible by 3 except for the case $G=\operatorname{Sz}\left(q^{r}\right)$. In that case the theorem would be trivially true. So we can suppose that 3 divides $|C|$. Reasoning as with $p=2$, we have that $C$ is 3-nilpotent. Then, taking into account earlier comments and Remark 2.9, the only possible case for $G$ is $\operatorname{PSL}\left(2,3^{r}\right)$ and $C$ is isomorphic to an intermediate subgroup between $\operatorname{PSL}(2,3) \cong A_{4}$ and its group of (inner-diagonal) automorphisms, $\operatorname{PGL}(2,3) \cong S_{4}$. The fact that $S_{4}$ is not 3 -nilpotent implies that $C \cong A_{4}$. In this case, we take $P$ an $A$ invariant Sylow 3-subgroup of $G$, which satisfies $\mathbf{N}_{G}(P)=P \rtimes C_{\frac{3^{r}-1}{2}}$ by Lemma 2.6(1). Also, by hypothesis, $\mathbf{N}_{G}(P)$ has an $A$-invariant 3-complement, say $H^{2}$, which is normalized by $\mathbf{N}_{C}(P)$. Since $C_{3} \cong P \cap C \leq \mathbf{N}_{C}(P)$, then $[H, P \cap C] \subseteq H \cap P=1$, that is, $P \cap C$ centralizes $H$. On the other hand, by computing orders it follows that $H$ is a Hall subgroup of $G$, so by taking any $A$-invariant Sylow subgroup of $H$ and applying Lemma 2.6(3), we conclude that $H$ cannot be centralized by $P \cap C$. This contradiction finishes the proof for $p=3$.

To end the proofs, as we mentioned in the introduction, we prove that the converse of Theorem B is true for every group. 
Corollary 3.3. Let $A$ be a group that acts on a group $G$ with $(|A|,|G|)=1$. Let $C:=$ $\mathbf{C}_{G}(A)$ and $p$ a prime. If $G$ has an $A$-invariant $p$-complement normalized by $C$, then for every A-invariant p-subgroup $1 \neq P$ of $G$, the group $\mathbf{N}_{G}(P)$ has an A-invariant $p$ complement normalized by $\mathbf{N}_{C}(P)$.

Proof. Let $1 \neq P$ be an $A$-invariant $p$-subgroup of $G$. By Theorem $A$, we have that $G$ is $p$-solvable, so $\mathbf{N}_{G}(P)$ is $p$-solvable too. Then, it possesses an $A$-invariant $p$-complement, say $K$. Now the $p$-solvability of $G$ and basic coprime action properties imply that $K$ lies in some an $A$-invariant $p$-complement $H$ of $G$, which is normalized by $C$ by hypothesis (in fact, $H$ is unique). As $K \leq H \cap \mathbf{N}_{G}(P)$, by order considerations, the equality holds. Since $C$ normalizes $H$, then $\mathbf{N}_{G}(P) \cap C$ normalizes $H \cap \mathbf{N}_{G}(P)=K$, as we wanted to prove.

We include some examples showing that Theorem B does not hold in general for primes different from 2 and 3.

Examples. Let $G=\mathrm{Sz}\left(2^{3}\right)$ acted on by the automorphism $\alpha$ of order 3 of the underlying field of 8 elements. Let $A=\langle\alpha\rangle$ and take $p=7$. Note that any non-trivial ( $A$-invariant) $p$-subgroup of $G$ is a Sylow subgroup, so we can use Lemma 2.7(2) to see that, for any such subgroup $P$, we have $\mathbf{N}_{G}(P)=D_{2 p}$ and it has an $A$-invariant $p$ complement $H$ (of order 2), which is trivially fixed by $A$ because $|A|$ is odd. It is trivially normalized by $\mathbf{N}_{C}(P)=H$. Thus, the hypothesis of Theorem $B$ is satisfied with $p=7$, however, $G$ has no ( $A$-invariant) 7-complements. We may use Lemma 2.7(3) and similar arguments to see that the same occurs in $G$ for $p=5$ and $p=13$.

Another example is $G=\operatorname{PSL}\left(2,2^{5}\right)$ with a field automorphism of order 5 inducing a coprime automorphism of $G$. The same arguments, but using Lemma 2.6(2) and (3) instead, show that Theorem B fails for $p=11$ and $p=31$.

\section{Acknowledgements}

The author would like to thank the referee for carefully reading the manuscript and for giving constructive comments which helped to improve the paper. This work is supported by Ministerio de Ciencia, Innovación y Universidades, Proyecto PGC2018-096872-B-100, by Universitat Jaume I, Proyecto UJI-B2019-03 and by Generalitat Valenciana, Proyecto $\mathrm{AICO} / 2020 / 298$.

\section{REFERENCES}

[1] A. Beltrán and G. Navarro. Actions and invariant character degrees III. J. Algebra 191 (1) (1997) 141-148.

[2] A. Beltrán and C.G. Shao. On the number of invariant Sylow subgroups under coprime action. J. Algebra 490 (2017) 380-389.

[3] J. H. Conway, R. T. Curtis, S. P. Norton, R. A. Parker, and R. A. Wilson. Atlas of Finite Groups. Oxford Univ. Press, Oxford, 1985.

[4] D. Gorenstein, R. Lyons, R. Solomon. The Classification of the Finite simple Groups, Number 3, vol. 40, American Math. Soc., Providence, 1998.

[5] R. M. Guralnick. Subgroups of prime power index in a simple group. J. Algebra 81 (2) (1983) 304-311.

[6] R. Hartley, Determination of the ternary collineation groups whose coefficients lie in the $\operatorname{GF}\left(2^{n}\right)$. Ann. of Math. 27 (1925) 140-158.

[7] B. Huppert, H. Blackburn. Finite groups II, III. Springer, Berlin, 1982. 
[8] B. Huppert. Endliche Gruppen I. Springer, Berlin, 1967.

[9] M.I. Isaacs. Finite Group Theory. Graduate Texts in Mathematics, vol. 92, American Mathematical Society, Providence, RI, 2008.

[10] M.Y. Kizmaz, On the influence of the fixed points of an automorphism to the structure of a group. J. Algebra, 572 (2021) 326-336.

[11] H. Kurzweil and B. Stellmacher. The theory of finite groups: An introduction. Springer-Verlag, Berlin-Heidelberg-New York, 2004.

[12] P. Mihăilescu. A class number free criterion for Catalan's conjecture. J. Number Theory, 99 (2003) 225-231.

[13] K. Zsigmondy. Zur Theorie der Potenzreste. Monatsh. Math. Phys. 3 (1892) 265-284. 